\title{
A Conversational Agent Framework using Semantic Analysis
}

\author{
Karen O'Shea, Zuhair Bandar, Keeley Crockett \\ Manchester Metropolitan University, United Kingdom
}

\begin{abstract}
This paper focuses on the implementation of a novel semantic-based Conversational Agent (CA) framework. Traditional CAs interpret scripts consisting of structural patterns of sentences. User input is matched against such patterns and an associated response is sent as output. This traditional CA approach, which solely takes into account the structural form of a sentence, requires the scripter to anticipate the inordinate ways that a user may send input. This is a tiresome and timeconsuming process. As such, a semantic-based CA that interprets scripts consisting of natural language sentences alleviates this burden by removing the process of pattern generation. The CA was evaluated by participants using a domain of a specific nature, that is, student debt management, which indicated promising results.
\end{abstract}

\section{Introduction}

A conversational agent is a computer program that can engage in conversation using natural language dialogue with a human participant. One of the earliest CAs developed was ELIZA [1]. By using a simple pattern matching technique, ELIZA was capable of creating the illusion that the system was actually listening to the user. There are two streams of CA development, that being, 'Embodied' CAs and 'Linguistic' CAs. Embodied CAs possess attributes such as an animated humanoid body and facial expressions, including movement of mouth and eye gaze. Much research is being focused on pursuing socially and emotionally aware CAs [2], [3]. Cassell et al. [3] cites that embodied CAs should now be capable of developing deeper relationships that make their collaboration with humans productive and satisfying over long periods of time.

ALICE [4] and InfoBot [5] are two text-based CAs able to extract data from a user, which may then be used throughout the conversation to demonstrate an element of awareness. Text-based CA's scripts are typically organized into contexts consisting of a number of hierarchically organized rules. Each rule possesses a list of structural patterns of sentences and an associated response. In some cases, text-based CAs are designed to work in a goal-oriented manner with the aim to achieving a specific goal. Infobot [5] is one such goal-oriented CA capable of interpreting structural patterns of sentences. An inordinate amount of scripting is required, however, in order to anticipate the many different ways a user may send an input string. Potentially large numbers of rules and patterns would be required. This is undoubtedly a time-consuming, high maintenance task. Secondly, modifying one rule or introducing a new rule into the script invariably has an impact on the remaining rules. As such, a reassessment of the entire script would be warranted, without which, would render the CA futile. The scripter is, therefore, required to remember the rankings of the rules and predict how the introduction of new rules will interact with existing rules [6]. The huge overhead and maintenance of this type of scripting undoubtedly suggests scope for an alternative approach. It has been highlighted that by employing sentence similarity measures, scripting can be reduced to a few prototype sentences [7], [8], [9], [10], [11].

Sentence similarity measures determine the semantic similarity of two sentences. Such measures can play an important role in conversational agent design. Two approaches to the measurement of sentence similarity are: 'Latent Semantic Analysis' (LSA) [12] and 'Sentence Similarity based on Semantic Nets and Corpus Statistics' [13]. LSA is a theory and method for extracting and representing the contextual-usage meaning of words by statistical computations applied to a large corpus of text [12]. A word by context matrix is formed based on the number of times a given word appears in a given set of contexts. The matrix is decomposed by "Singular Value Decomposition" (SVD) into the product of three other matrices, including the diagonal matrix of singular values [12]. This dimension reduction step collapses the component matrices so that words that occurred or did not occur in some contexts now appear with a greater or lesser frequency [12]. Reconstruction of the original matrix enables LSA to acquire word knowledge among large numbers of contexts. Although LSA makes no use of syntactic relations, it does, however, offer close enough approximations of people's knowledge to underwrite 
and test theories of cognition. LSA is suited to substantial sections of text as opposed to single sentences. Sentence Similarity based on Semantic Nets and Corpus Statistics overcomes these limitations by forming word vectors dynamically based entirely on the words in the compared sentences. The measure also considers a further aspect of primary syntactical information in the form of word order. As such, this was selected as the measure to be employed within the semantic-based CA framework for the purposes of this work due to its application to texts of sentence length. This measure will be subsequently described in further detail in section 2 .

This paper will present the development of a semantic-based CA framework using goal-oriented dialogue. By using such a framework, scripting will be greatly reduced and simplified in contrast to that of the high overheads of pattern-scripted CA approaches and their maintenance. This underlies the benefits of a CA that interprets word meaning as opposed to its structural form.

The paper is organized as follows: section 2 will describe and illustrate the sentence similarity measure; section 3 will describe the implementation of a semantic-based CA framework; section 4 will describe the experimental methodology; section 5 will present the results and discussion and section 6 will conclude and highlight areas for further work.

\section{Sentence similarity measure}

The sentence similarity measure [13] based on Semantic Nets and Corpus statistics focuses directly on computing the similarity between very short texts of sentence length. Through the use of a lexical/semantic knowledge-base, such as WordNet [14], the length of separation between two words can be measured, which in turn, can be used to determine word similarity. The meeting point of their path is known as the subsumer, which is similarly measured by counting the levels to the top of the hierarchy. Li et al. [12], [15] proposed that the similarity between two words be a function of the attributes: path length and depth. To understand the algorithm, the following example is used. Taking two sentences (T1 and T2) a joint word set is formed using only distinct words. For example:

$\mathrm{T} 1=$ Mars is a small red planet

T2 $=$ Mars and Earth orbit the sun

A joint word set ' $\mathrm{T}$ ' is formed where:

$\mathrm{T}=$ Mars is a small red planet and earth orbit the sun

As a result, each sentence is represented by the use of the joint word set with no surplus information. Raw semantic vectors are then derived for each sentence using the hierarchical knowledge-base WordNet, in order to determine the separation between the words. Taking a non-linear transfer function as an appropriate measure, the following formula denotes a monotonically decreasing function of $l$, where $l=$ path length between words and $\alpha$ is a constant.

$f(l)=e^{-\alpha l}$

As for the depth of the subsumer, the relationship of words at varying levels of the hierarchy must be taken into consideration. For example, words at the upper layers are far more general and less semantically similar than words at lower layers [13]. Therefore, subsuming words at upper layers must be scaled down whereas words at lower layers must be scaled up, resulting in a monotonically increasing function of $h$, where $h=$ depth of subsumer and $\beta$ is a constant.

$f(h)=\left(e^{\beta l}-e^{-\beta h}\right) /\left(e^{\beta l}+e^{-\beta h}\right)$

As such, the raw similarity $s(w 1, w 2)$ between two words is calculated as:

$s(w 1, w 2)=e^{-\alpha l} \cdot\left(e^{\beta l}-e^{-\beta h}\right) /\left(e^{\beta l}+e^{-\beta h}\right)$

where $\alpha=0.2$ and $\beta=0.45$.

Each word is then weighted, ie. assigned an information content value, based on its significance and contribution to contextual information. By combining the raw semantic vector $s(w 1, w 2)$ with the information content of each word, $I(w l)$ and $I(w 2)$, semantic vectors are created:

$s_{i}=s(w 1, w 2) . I(w 1) \cdot I(w 2)$

Finally, the semantic similarity $S s$ between two sentences, $s 1$ and $s 2$, is calculated as:

$$
\text { Ss }=\operatorname{si1} . s i 2 / \sqrt{s i 1} / \sqrt{s i 2}
$$

where si 1 is the resultant semantic vector of sentence 1 and $s i 2$ is the resultant semantic vector of sentence 2.

Word order also plays an active role in sentence similarity. Each word is assigned a unique index number which simply represents the order in which the word appears in the sentence. For example, take the following sentences denoted T1 and T2:

$\mathrm{T} 1=$ The cat ran after the mouse

$\mathrm{T} 2=$ The mouse ran after the cat

A joint word set ' $\mathrm{T}$ ' is formed where:

$\mathrm{T}=$ The cat ran after the mouse 
Each sentence is then compared to that of the joint word set. If the same word is present - or if not, the next most similar word - then the corresponding index number from $\mathrm{T} 1$ will be placed in the vector, r1. As such, the word order vectors $r 1$ and $r 2$ for the example sentence pair $\mathrm{T} 1$ and $\mathrm{T} 2$ would be formed as follows:

$\mathrm{r} 1=\{123456\}$

$\mathrm{r} 2=\{163452\}$

Therefore, word order similarity $\mathrm{Sr}$ is calculated as:

$$
S r=1-\sqrt{(r 1-r 2)} / \sqrt{(r 1-r 2)}
$$

Finally, the sentence similarity is derived by combining both semantic similarity and word order similarity. The overall sentence similarity between two sentences $S(T 1, T 2)$ is calculated as:

$$
S(T 1, T 2)=\delta S s+(1-\delta) S r
$$

$\delta$ takes into account that word order plays a less significant role when determining sentence similarity.

\section{Semantic-based conversational agent}

This section will describe the proposed semanticbased CA framework and its novel natural language scripting methodology. This will be followed by the application of the framework and its associated features as a semantic-based CA.

\subsection{Framework overview}

The proposed semantic-based CA framework uses semantics as a means to measure sentence similarity. As such, scripts maybe composed of prototype natural language sentences as opposed to structural patterns of sentences used by traditional approaches. In the semantic-based framework, the CA is organized into contexts consisting of a number of similarly related rules. Through the use of a sentence similarity measure [11], a match is determined between the user's utterance and the scripted natural language sentences. Similarity ratings are measured in the range from 0 to 1 in which a value of 0 denotes no semantic similarity and 1 denotes an identical sentence pair. The highest ranked sentence is fired and its associated response is sent as output. This is true, however, if the firing strength is above that of a pre-determined threshold, otherwise an associated default response is returned. A threshold is required in order to ensure that sufficient similarity has been found. Determination of an appropriate threshold is, therefore, found through experimentation. The determined threshold value is sufficiently low enough to capture semantically similar sentence pairs but sufficiently high enough to filter out noise, that being, sentences unrelated to the context.

The scripts are written to enable the semanticbased CA to operate in a goal-oriented manner, that being, to achieve a specific aim during dialogue. The scripts will be structured using a contextual knowledge pathway (CKP). This will be discussed further in section 3.2.

The components of the semantic-based CA framework are shown in figure 1. Each component will now be described.

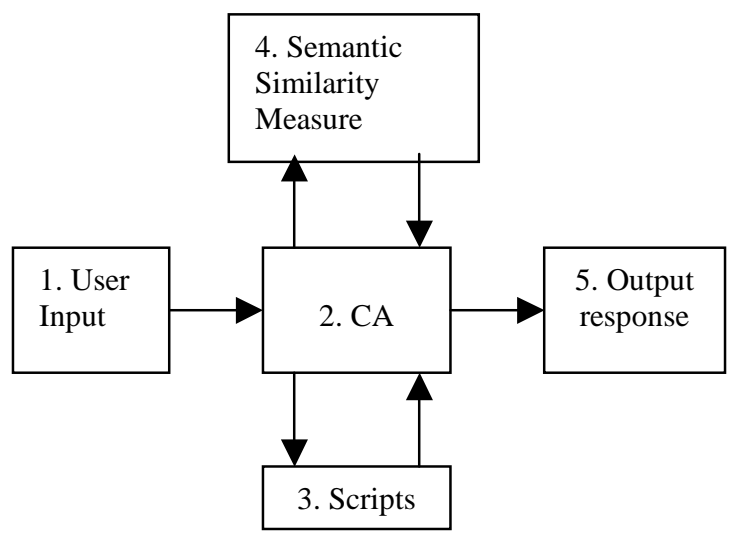

Figure 1. CA framework

1. Natural language dialogue is received as input from the user.

2. Semantic-based CA receives natural language dialogue from the user that is sent to the sentence similarity measure.

3. Semantic-based $\mathrm{CA}$ receives natural language sentences from the scripts files that are sent to the sentence similarity measure.

4. Sentence similarity measure calculates a firing strength for each sentence pair that is returned and processed by the semanticbased CA.

5. The highest ranked sentence is fired and its associated response is sent as output.

\subsection{Scripts}

Scripts consist of contexts that relate to a specific theme or topic of conversation. Each context contains one or more rules, which possess a number of prototype natural language sentences. A scripted natural language rule is shown below where ' $\mathrm{s}$ ' is a natural language sentence and ' $r$ ' is a response statement. 
$<$ Rule_01>

s: I have no money

r: I'm sorry to hear that

A pattern-scripted rule is shown below where ' $p$ ' is a structural pattern of a sentence and ' $r$ ' is a response statement.

$<$ Rule_01>

p: * money

$\mathrm{p}: *$ cash

p: * dosh

p: * funding

r: I'm sorry to hear that

When comparing the above two rule structures, the obvious benefits are apparent. When constructing the pattern-scripted rule, every permutation of that sentence is required. In contrast, the scripted natural language rule required one such sentence in this particular case. Interpreting word meaning as opposed to the use of a pattern-matching technique means that rule structures are simplified and reduced in size and complexity.

The structure of the scripts will be described using a new technique known as a contextual knowledge pathway (CKP) structured to work, in this particular case, in a goal-oriented manner. That is, in this particular case, the contexts are arranged in an order that a user must traverse in order to reach goal fulfillment. That is, to achieve a satisfactory answer to a question/query. Users may switch between contexts, traversing forwards or backwards along the CKP. As such, the contexts along the CKP express specific queries, which require specific answers in order for progression to be made along one of possibly various routes. Users may switch between contexts, traversing forwards or backwards along the CKP. Engaging in dialogue with a user, the CA is able to capture specific pieces of information from the user input in order to progress along the network of contexts described by the CKP.

Using a goal-oriented CKP, the aim is to elicit a specific set of answers from the user in order to achieve goal-fulfillment. As such, rules that pose a question possess a series of successor rules, which seek to obtain an appropriate answer. If no relevant input is sent, that is, no match found above that of the threshold, an associated default rule is returned prompting the user for further input to satisfy the request.

Each rule is assigned an activation level of one. Once a rule has fired its activation level is reduced to zero and as such will fail to fire again from subsequent user input. For example, if a rule has performed its task of eliciting a piece of information from the user, its purpose is no longer required.

For the purposes of this work, the scripts are focused on one domain purely to demonstrate the semantic-based CA framework. The selected domain is concerned with advising students at university on debt management and the payment of tuition fees. The CKP will contain seven interconnected contexts, as illustrated in figure 2. Entities representing the design of the CKP are described in table 1.

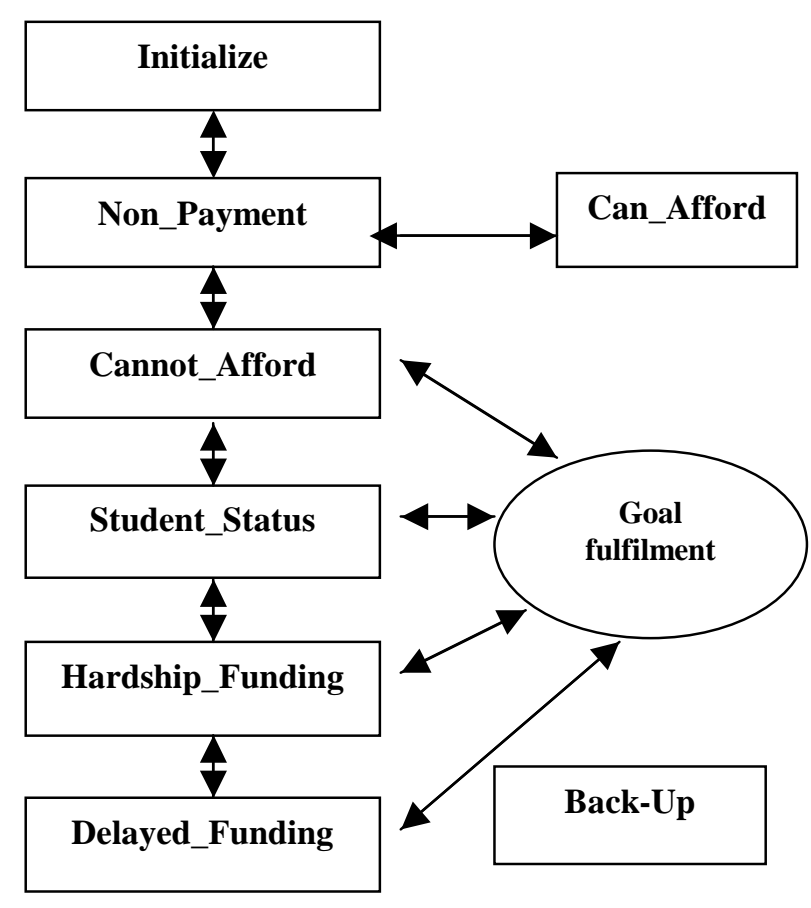

Fig. 2. Contextual Knowledge Pathway (CKP) 


\section{Table 1. Entities of a contextual knowledge pathway representation}

\begin{tabular}{|l|l|}
\hline \multicolumn{1}{|c|}{ Entity } & \multicolumn{1}{|c|}{ Description } \\
\hline & $\begin{array}{l}\text { Context representation } \\
\text { consisting of rule } \\
\text { structures specific to that } \\
\text { context. }\end{array}$ \\
\hline & $\begin{array}{l}\text { Flow of user input from } \\
\text { one context to the next. } \\
\text { Dialogue may move in } \\
\text { various directions en } \\
\text { route to goal-fulfilment. }\end{array}$ \\
\hline & $\begin{array}{l}\text { A depiction of goal- } \\
\text { fulfillment. A point } \\
\text { where the user will have } \\
\text { achieved a satisfactory } \\
\text { answer to their initial } \\
\text { enquiry. There is an } \\
\text { option to return to } \\
\text { dialogue if, however, a } \\
\text { miss-interpretation has } \\
\text { occurred. }\end{array}$ \\
\hline
\end{tabular}

The contexts along the CKP express specific queries, which require specific answers in order for progression to be made along the designated route. Dialogue will traverse the CKP in a progression starting with the base context named 'Initialize'. It is possible to revert back to a previously visited context in the case of a miss-interpreted line of input. As such, the user has the option to alert the CA that there has been a miss-understanding. The following contexts: 'Non_Payment' aims to elicit the reason for non-payment of the tuition fees; 'Can_Afford' identifies that the student has in fact paid the fees and as such reached goal-fulfillment; 'Cannot_Afford' aims to elicit why a student simply cannot afford even to pay the proposed one third of the debt; 'Student_Status' identifies the status of the student, for example, a 'Home' student or 'International' student; 'Hardship_Funding' determines whether the student has applied for such funding and 'Delayed_Funding' aims to identify if the funding has been delayed. The 'Back-Up' context is one in which the CA will revert to if no content within the current context is appropriate to that of the user input.

\section{Experimental methodology}

This section will review the selected domain of the goal-oriented CKP. This will follow a description of the experiments used as a means to evaluate the framework.

\subsection{Domain}

The domain used to script the semantic-based CA is concerned with advising students at university on debt management and the payment of tuition fees. The dialogue must traverse the CKP in order to achieve goal-fulfillment. The CKP consists of contexts based on previous analysis on why students fail/delay to pay their tuition fees [16]. The CA, namely Sam, is therefore designed to identify the reason why a student has not paid and offer advice and guidance on how to find ways to pay the fees.

\subsection{Initial experiments}

Experiments were undertaken to evaluate the semantic-based CA from a subjective viewpoint. Three individuals were asked to converse with the semantic-based CA each using a pre-determined domain-specific scenario.

Each individual was asked to converse with Sam in natural language based closely as possible to the scenario. The scenario is described as follows: User must declare that he/she is unable to pay for their fees, due in part, to the lack of sponsorship money or the loss of employment. He/she is unable to state their student status, that being, 'Home' or 'International' but later resort to being Scottish. The user is in their final year of study. User is able to ask questions relevant to the domain during dialogue and seek clarification.

The CKP guides the user during the conversation with the aim of achieving goal-fulfillment. If Sam is unable to match any user input to the scripted natural language sentences, a default response is generated with the aim to keep the user on track and prompt them for further input. Sam captures information during dialogue and may ask for clarification if required.

\section{Results and discussion}

The design of the experiment entailed three individuals to converse with the semantic-based CA using one pre-determined scenario. The transcript of dialogue one is shown in figure 3 . 
Sam: Hello my name is Sam. Please tell me why you have not paid your tuition fees?

Tom: I have not paid

Sam: OK so you have not paid. The only other options are that you have withdrawn or there is some other reason.

Tom: I don't have the funds to pay

Sam: Sorry, I didn't quite understand that. Please tell me why you have not paid your fees before we move on. If you don't know, then say so, and I will run through some options.

Tom: I can't afford to pay

Sam: OK I may have a solution. Can you pay one third of the debt within the next week?

Tom: I will pay a third in the next month

Sam: Right so you can afford a third. Tell me are you a Home student?

Tom: I am not an international student

Sam: OK so you are a Home student. So my next question has to be are you in your final year of study?

Tom: I am in my second year

Sam: Well you have left it too late. You must pay the remainder of your fees as soon as possible. If you have no further questions please type bye to end the session.

Tom: Bye

\section{Fig. 3. Dialogue one}

The user input and fired sentence pairs for dialogue one are displayed in table 2 .

\section{Table 2. User input and fired sentence pairs for dialogue three}

\begin{tabular}{|l|l|}
\hline \multicolumn{1}{|c|}{ User Input } & \multicolumn{1}{c|}{ Fired Sentence } \\
\hline 1. I have not paid & I've not paid \\
\hline $\begin{array}{l}\text { 2. I don't have the } \\
\text { funds to pay }\end{array}$ & $\begin{array}{l}\text { No appropriate match } \\
\text { found - Default fired }\end{array}$ \\
\hline 3. I can't afford to pay & I can not afford it \\
\hline $\begin{array}{l}\text { 4. I will pay a third } \\
\text { next month }\end{array}$ & $\begin{array}{l}\text { I can afford a third in } \\
\text { the next seven days }\end{array}$ \\
\hline $\begin{array}{l}\text { 5. I am not an } \\
\text { international student }\end{array}$ & $\begin{array}{l}\text { I' m not an } \\
\text { international student }\end{array}$ \\
\hline $\begin{array}{l}\text { 6. I am in my second } \\
\text { year }\end{array}$ & I am in my final year \\
\hline
\end{tabular}

The results of the user input/fired sentence pairs are as follows: The outputs generated after the input of user utterances 1, 3, and 5 indicate a correct firing. Sufficient semantic similarity between the user utterances and the corresponding natural language sentences was calculated and an appropriate response was returned.
The outputs generated after the input of user utterance 4 and 6 indicate a miss-firing. However sufficient semantic similarity was identified for the sentence pairs to fire. As such, an inappropriate response was returned as output. The miss-firing of a sentence pair, that being, an inappropriate or unexpected match, can be explained as follows. Take, for example, user input and fired sentence pair \#4 in table 2.

User input: I will pay a third next month Fired sentence: I can afford a third in next seven days

To re-iterate the aforementioned sentence similarity measure initializes by forming a joint word set, in this case, 'I will pay a third next month can afford seven days', whereby only distinct words are used, thereby containing no surplus information. The joint word set is compared to both the user input and the scripted natural language sentences through the use of the hierarchical knowledge-base, WordNet. For example, table 3 displays the resultant semantic vector for the user input 'I will pay a third next month' against that of the joint word set.

\section{Table 3. Resultant semantic vector for user input \#4}

\begin{tabular}{|l|l|l|l|l|l|l|l|}
\hline & I & will & pay & a & third & next & month \\
\hline I & 1 & & & & & & \\
\hline Will & & 1 & & & & & \\
\hline Pay & & & 1 & & & & \\
\hline A & & & & 1 & & & \\
\hline Third & & & & & 1 & & \\
\hline In & & & & & & & \\
\hline The & & & & & & & \\
\hline Next & & & & & & 1 & \\
\hline Month & & & & & & & 1 \\
\hline Can & & & & & 0.5091 & & \\
\hline Afford & & & & & & & \\
\hline Seven & & & & & & & \\
\hline days & & & & & & & 0.7829 \\
\hline \multicolumn{1}{|c|}{$\boldsymbol{s}$} & & & & & 0.5091 & & 0.7829 \\
\hline $\begin{array}{l}\text { Resultant } \\
\text { measure }\end{array}$ & & & & & 0.5828 & & 0.8155 \\
\hline
\end{tabular}

In table 3, the first row denotes the joint word set and the first column denotes the user input string. Each word in the joint words set is matched against each word in the user input string. Identically matched words are assigned a value of 1 in the cell at the cross point. All other word pairs deeming to possess sufficient semantic similarity are included if above that of the word pair similarity threshold. For example, the word 'can' does not appear in the user input string, but the most similar word is 'third', with a word pair similarity of 0.26326 . Thus, the cell at the cross point of 'can' and 'third' is set to 0.26326 . 
Incidentally, the word pair 'can' and 'third' could be deemed as an unexpected relation with respect to context. To illustrate the semantic relation, the paths of the target word pairs are traced using the hierarchical knowledge-base of WordNet, shown in figure 4 .

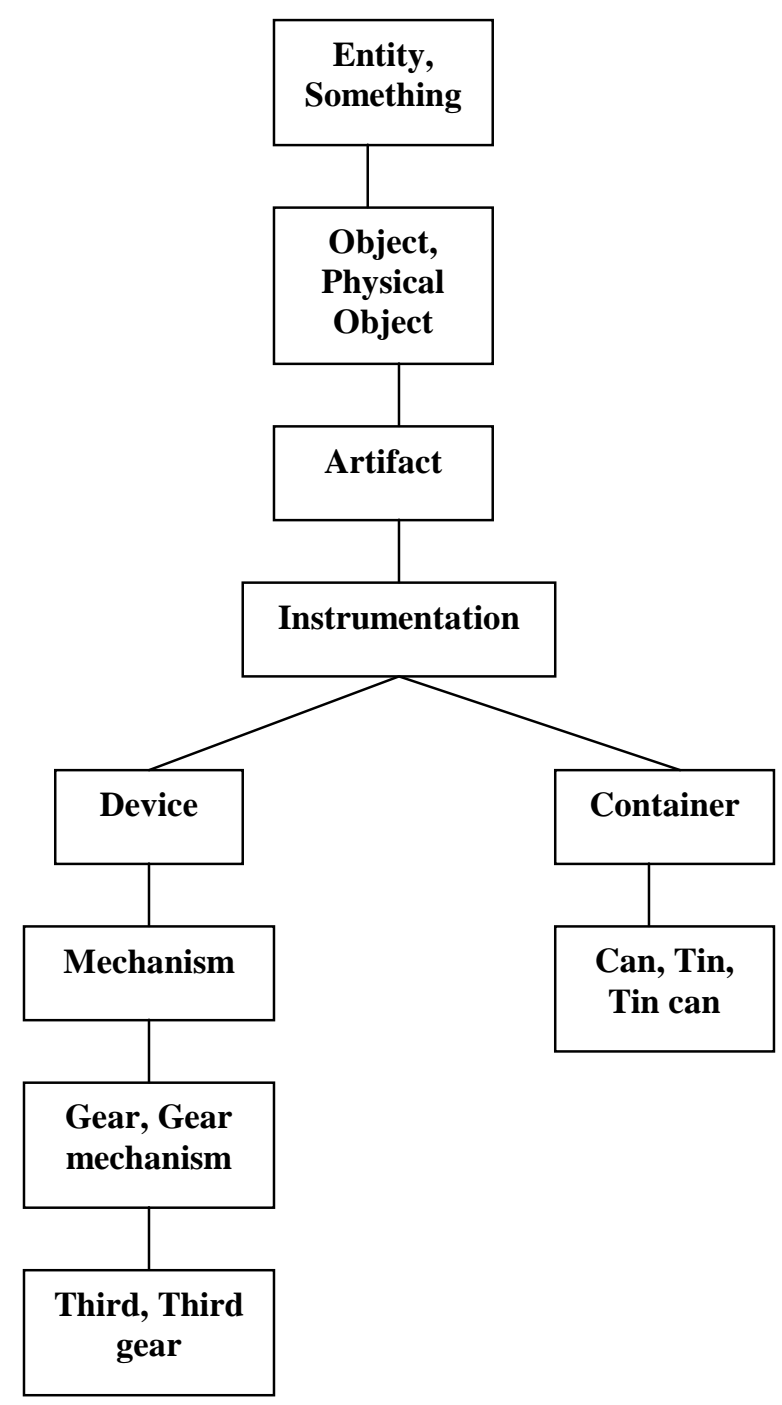

Fig. 4. Semantic path separation

Words may be polysemous, that being, possess multiple meanings. The target words 'third' and 'can' both possess six senses. The aim, however, of the sentence similarity measure is to identify the two senses of each word with the shortest length of separation. In this case, 'third' as in the sense of a driving mechanism and 'can' as in the sense of a tin can container. As such, the separation of six synsets results in a semantic word pair measure above that of the word pair similarity threshold. As a consequence, inappropriate senses of the target words were used, which ultimately impacted on the rating of the sentence pair and, thus, resulted in a miss-firing. A more appropriate selection of sense would have been 'third' as in a 'fraction of a measure'. The word 'can' is, however, treated as a verb in this particular context. As such, there is no apparent semantic relation between the word pairs. Understanding context within a sentence structure and the use of parts-of-speech other than that of nouns are outside the scope of this sentence similarity measure.

One expected relation is observed between the word pairs 'days' and 'month' producing a word pair similarity of 0.48149 . Accumulation of the resultant semantic word pair measures results in an overall semantic similarity value of 0.5375 . A contribution of word order similarity of 0.7362 produces an overall sentence similarity rating of 0.5673 . A rating of which is above that of the sentence similarity threshold of 0.3800 . However, due to the fact that the prior output response asked 'Can you pay one third of the debt within the next week?' one can subjectively view this as a miss-fired sentence pair.

The outputs generated after the input of user utterance \#2, shown in table2, indicate that there was insufficient semantic similarity between the user utterance and the scripted natural language sentences. As such, an appropriate default was sent as output to prompt the user for further input. The transcript of dialogue two is shown in figure 5. 
Sam: Hello my name is Sam. Please tell me why you have not paid your tuition fees?

Tom: I haven't got it

Sam: Well if you have not withdrawn that leaves paid or some other reason.

Tom: I haven't paid as I have no money

Sam: OK so you have not paid and you have not withdrawn. Go ahead and tell me why you have not paid your debt.

Tom: I don't have the money

Sam: OK I may have a solution for you. Can you pay a third of the debt within the next week.

Tom: no I told you I have no money left

Sam: Sorry, I didn't quite understand that. Please answer the question. Can you afford to pay one third of the debt by next week.

Tom: not really I have spent it

Sam: Sorry, I didn't quite understand that. Please answer the question. If I have to repeat myself again, I will have to end the session. Can you afford to pay one third of the debt by next week.

Tom: OK

Sam: OK first of all, tell me, are you a Home student.

Tom: What does that mean.

Sam: British citizens are home students and EU citizens count under European agreements. Visitors from other countries usually don't count as Home students. So try and answer my question.

Tom: I don't know

Sam: Don't let this question worry you. There are different ways of supporting Home students and International students. I therefore need a clear and definite answer from you.

Tom: I'm Scottish

Sam: OK so you are a Home student. So my next question has to be, are you in your final year?

Tom: no

Sam: Well then you could pay it off in three equal instalments. You will need to send an email to credit control. Please type bye if you have no further questions.

Tom: How much do I have to pay

Sam: The payments are done in three equal instalments. Go to your faulty office to arrange it.

Tom: Bye

\section{Fig. 5. Dialogue two}

The user input and fired sentence pairs for dialogue two are displayed in table 4.
Table 4. User input and fired sentence pairs for dialogue two

\begin{tabular}{|l|l|}
\hline \multicolumn{1}{|c|}{ User Input } & \multicolumn{1}{c|}{ Fired Sentence } \\
\hline 1. I haven't got it & I haven't left \\
\hline $\begin{array}{l}\text { 2. I haven't paid as I } \\
\text { have no money }\end{array}$ & I haven't paid \\
\hline $\begin{array}{l}\text { 3. I don't have the } \\
\text { money }\end{array}$ & $\begin{array}{l}\text { I don't have the money } \\
\text { to pay }\end{array}$ \\
\hline $\begin{array}{l}\text { 4. no I told you I have } \\
\text { no money left }\end{array}$ & $\begin{array}{l}\text { No appropriate match } \\
\text { found - Default fired }\end{array}$ \\
\hline $\begin{array}{l}\text { 5. not really I have } \\
\text { spent it }\end{array}$ & $\begin{array}{l}\text { No appropriate match } \\
\text { found - Default fired }\end{array}$ \\
\hline 6. ok & OK I can pay a third \\
\hline $\begin{array}{l}\text { 7. what does that } \\
\text { mean }\end{array}$ & What do you mean \\
\hline 8. I don't know & I don't know \\
\hline 9. I'm Scottish & I am scottish \\
\hline 10. no & no \\
\hline $\begin{array}{l}\text { 11. how much do I } \\
\text { pay for each } \\
\text { installment }\end{array}$ & $\begin{array}{l}\text { How much are the } \\
\text { installments }\end{array}$ \\
\hline
\end{tabular}

The results of the user input/fired sentence pairs are as follows: The outputs generated after the input of user utterances $2,3,6,7,8,9,10$, and 11 indicate a correct firing. As a result, the semantic-based CA identified sufficient semantic similarity between the user input and the scripted natural language sentences.

The outputs generated after user input \#1 indicate a miss-firing. As a result, the semantic-based CA failed to identify sufficient semantic similarity between the user input and the scripted natural language sentences. This resulted in a miss-match and the firing of an inappropriate rule.

The outputs generated after user input 4 and 5 indicate that there was insufficient semantic similarity found between the user utterance and the scripted natural language sentences. As such, an appropriate default response was sent as output to prompt the user for further input.

The transcript of dialogue three is shown in figure 6. 
Sam: Hello my name is Sam. Please tell me why you have not paid your tuition fees?

Tom: I'm not sure

Sam: Well if you have not paid or you have not withdrawn then the only other option is that there is some other reason.

Tom: ok can you help me

Sam: Sorry, I didn't quite understand that. Please answer the question. If you have not paid or you have not withdrawn then the only other option is that there is some other reason.

Tom: ok it's another reason

Sam: OK so you have not paid and you have not withdrawn. Go ahead and tell me why you have not paid your debt.

Sam: OK so you have not paid and you have not withdrawn. Go ahead and tell me why you have not paid your debt.

Tom: I didn't have the money after paying the rent.

Sam: Sorry, I didn't quite understand that. Please tell me why you have not paid your fees before we move on. If you don't know, then say so, and I will run through some options.

Tom: I don't know

Sam: Well the reasons are typically: you have student loan problems, a sponsorship problem or you simply can't afford it.

Tom: can't afford it

Sam: OK I may have a solution. Can you pay one third of the debt within the next week?

Tom: I can afford a third within the next seven days

Sam: Right so you can afford a third. Tell me are you a Home student

Tom: Perhaps

Sam: I need a definite answer from you. Are you a Home student?

Tom: I am from Scotland

Sam: OK so we have established something about your financial situation. So my next question has to be are you in your final year of study?

Tom: no

Sam: Well then you could pay it off in three equal installments. You will need to send an email to credit control. Please type bye if you have no further questions.

Tom: Bye

\section{Fig. 6. Dialogue three}

The user input and fired sentence pairs for dialogue three are displayed in Table 5.
Table 5. User input and fired sentence pairs for dialogue three

\begin{tabular}{|l|l|}
\hline \multicolumn{1}{|c|}{ User Input } & \multicolumn{1}{c|}{ Fired Sentence } \\
\hline 1. I'm not sure & I am not sure \\
\hline 2. ok can you help me & $\begin{array}{l}\text { No appropriate match } \\
\text { found - Default fired }\end{array}$ \\
\hline $\begin{array}{l}\text { 3. ok it's another } \\
\text { reason }\end{array}$ & Some other reason \\
\hline $\begin{array}{l}\text { 4. I didn't have the } \\
\text { money after paying } \\
\text { the rent }\end{array}$ & $\begin{array}{l}\text { No appropriate match } \\
\text { found - Default fired }\end{array}$ \\
\hline 5. I don't know & I don't know \\
\hline 6. can't afford it & I can't afford to pay \\
\hline $\begin{array}{l}\text { 7. I can afford a third } \\
\text { within the next seven } \\
\text { days }\end{array}$ & $\begin{array}{l}\text { I can afford to pay a } \\
\text { third of the debt within } \\
\text { the next seven days }\end{array}$ \\
\hline 8. perhaps & Perhaps I am \\
\hline 9. I am from Scotland & I am Scottish \\
\hline 10. no & no \\
\hline
\end{tabular}

The results of the user input/fired sentence pairs are as follows: The outputs generated after the input of user utterances $1,3,5,6,7,8,9$ and 10 indicate a correct firing. This again highlights that a semantic match was identified above that of the threshold.

The outputs generated after the input of user utterances 2 and 4 indicate that no match was found above that of the threshold between the user input and the scripted natural language sentences. As such, an appropriate default was sent as output to prompt the user for further input.

To conclude, in all cases that resulted in a missmatch or the firing of a default rule, were due to a lack of relevant prototype natural language sentences. This, however, may be rectified by incorporating additional natural language sentences to the rules to account for these permutations.

\section{Conclusions and further work}

This paper was concerned with the development of a semantic-based CA framework. Via the use of a sentence similarity, scripting is reduced to a select number of natural language sentences. This is in contrast with traditional pattern-scripted CAs that required potentially large numbers of patterns.

The results indicate that a CA based solely on semantic similarity can fair well when subjected to natural language input. This is based on the scripting of a representative sample of prototype sentences, which is decided through experimentation.

Further work will entail an evaluation with human participants using scenarios with more depth and challenge. It is proposed that the results will be compared to that of a pattern-scripted CA based on the same domain. Current work focuses on the development of memory attributes to add character 
and self-awareness. Additionally, due to the use of natural language scripting, automatic rule generation, to an extent, will be developed. This alleviates the burden of generating new rules. The process will involve the automatic generation of a new rule possessing the unmatched user input string. A human scripted would then be required to add an appropriate response to complete the rule structure. This feature would prove difficult using a pattern-scripted CA approach as this would involve the generation of patterns - a process achieved through human intuition.

\section{References}

[1] J. Weizenbaum, ELIZA - “A Computer Program for the Study of Natural Language Communication between Man and Machine", Communications of the Association for Computing Machinery, Vol. 9, 1966, pp. 36-45.

[2] T. Bickmore and J. Cassell, "How about this weather?" Social Dialog with Embodied Conversational Agents. Fall Symposium on "Narrative Intelligence", Cape Cod, MA, 2000, pp. 48.

[3] J. Cassell, A. Gill, P. Tepper, Coordination in Conversation and Rapport. Workshop on Embodied Natural language, Association of Computationl Linguistics. Prague, 2007.

[4] R. S. Wallace. (2008, February 01). ALICE: Artificial Intelligence Foundation Inc. [Online]. Available: http://www.alicebot.org. Accessed: March 7, 2009.

[5] D. Michie, and C. Sammut, Infochat ${ }^{\mathrm{TM}}$ Scripter's Manual. Manchester: Convagent Ltd, 2001.

[6] C. Sammut, "Managing Context in a Conversational Agent", Electronic Transactions on Artificial Intelligence, Vol. 3 (7), 2001, pp. 1-7.

[7] J. O'Shea, Z. Bandar, K. Crockett, D. Mclean, “A Comparative Study of Two Short Text Semantic Similarity Measures", Lecture Notes on Artificial Intelligence, Vol. 4953, pp. 172, Springer, 2008.

[8] Y. Li, "AMethod for Measuring Sentence Similarity and its Application to Conversational Agents", FLAIRS Conference, 2004.

[9] K.O'Shea, Z. Bandar, K. Crockett, “A Novel Approach for Constructing Conversational Agents using Sentence Similarity Measures", World Congress on Engineering, International Conference on Data Mining and Knowledge Engineering, London, 2008, pp. 321-326.
[10] K.O’Shea, Z. Bandar, K. Crockett, “Towards a New Generation of Conversational Agents using Sentence Similarity" in Advances in Electrical Engineering and Computational Science, Lecture Notes in Electrical Engineering, Vol 39, Sio-Long Ao and Len Gelman, Ed. Netherlands: Springer, 2009, pp. 505514.

[11] K.O'Shea, Z. Bandar, K. Crockett, "A SemanticBased Conversational Agent Framework", The $4^{\text {th }}$ International Conference for Internet Technology and Secured Transactions (ICITST-2009), Technical CoSponsored by IEEE UK?RI Communications Chapter, London, 9-12 November 2009, pp. 92-99.

[12] T. K. Landauer, P. W. Foltz, and D. Laham, "Introduction to Latent Semantic Analysis". Discourse Processes, Vol. 25 (2-3), 1998, pp. 259284.

[13] Y. Li, D. McLean, Z. A. Bandar, J. D. O'Shea, and K. Crockett, "Sentence Similarity Based on Semantic Nets and Corpus Statistics", IEEE Transactions on Knowledge and Data Engineering, Vol. 18, (8), 2006, pp. 1138-1149.

[14] G. A. Miller, "WordNet: A Lexical Database for English", Comm. ACM, Vol. 38, no. 11, 1995, pp. $39-41$.

[15] Y. Li, Z. A. Bandar, and D. Mclean, "An Approach for Measuring Semantic Similarity between Words using Multiple Information Sources", IEEE Transactions on Knowledge and Data Engineering, Vol. 15, (4), 2003, pp. 871-881.

[16] Convagent Ltd, available: www.convagent.com. Accessed: March 7, 2009. 